\title{
Validez de la versión española de la Hypersensitive Narcissism Scale (HSNS) en una Unidad de Conductas Adictivas
}

\author{
Validity of the Spanish version of the Hypersensitive \\ Narcissism Scale (HSNS) in subjects attended at an \\ Addiction Treatment Unit
}

| Carmen RipolL*; José Salazar ${ }^{\star \star} ;$ Julio Bobes ${ }^{\star \star \star}$
* Médico de Conductas Adictivas. Unidad de Conductas Adictivas de Campanar. Departamento de Salud 7. Conselleria de Sanitat. Generalitat Valenciana.

** Psiquiatra. Centro de Salud Mental de Paterna. Departamento de Salud 6. Conselleria de Sanitat. Generalitat Valenciana. Centro de Investigación Biomédica en Red de Salud Mental (CIIBERSAM) Salud Mental.

*** Catedrático de Psiquiatría. Área de Psiquiatría - Universidad de Oviedo. Centro de Investigación Biomédica en Red de Salud Mental (CIBERSAM).

Enviar correspondencia a:

José Salazar. USM Paterna. Clot de Joan s/n. Paterna (Valencia).

E-mail: j.salazar@wanadoo.es.

\section{RESUMEN}

Introducción. La personalidad narcisista representa un componente importante de los trastornos de personalidad en los sujetos con abuso o dependencia de drogas y aunque existen validaciones de instrumentos de evaluación de narcisismo manifiesto y autoestima, no se ha validado hasta ahora ninguna prueba de valoración del narcisismo hipersensible en lengua española. Objetivo. Comprobar la validez de la versión española de la escala de narcisismo encubierto Hypersensitive Narcissism Scale (HSNS) en la población indicada.

Método. En una muestra de 79 pacientes se evaluó la fiabilidad mediante el alfa de Cronbach y el coeficiente de correlación intraclase (CCIC), la validez de constructo mediante análisis factorial, y la validez concurrente mediante la correlación de la HSNS con medidas de gravedad, discapacidad, autoestima, narcisismo manifiesto o trastornos de personalidad. Resultados. La fiabilidad de la puntuación total de la escala resultó satisfactoria (alfa de Cronbach $=0,73 ; \mathrm{CCIC}=0,67$ ), aunque algunos items precisarán su reconsideración. El análisis factorial mostró buena validez de constructo mediante tres factores compatibles con la teoria. Respecto a la validez concurrente, el narcisismo encubierto (HSNS) se correlacionó positivamente con el narcisismo manifiesto, la gravedad y la discapacidad debidas al consumo de drogas, y negativamente con la autoestima. Los valores más altos de narcisismo encubierto se manifestaron en los trastornos de personalidad límite, narcisista y pasivo-agresivo. Conclusiones. La versión española de la HSNS puede resultar un instrumento válido para la estimación del narcisismo encubierto en sujetos tratados por adicción o abuso de substancias..

Palabras clave: narcisismo, narcisismo encubierto, HSNS, trastorno de personalidad, abuso de sustancias.

\section{ABSTRACT}

Introduction. Narcissistic personality is an important component of personality disorders which are prevalent in those presenting drug abuse or dependence. Assessment instruments usually consider self-esteem, narcissism and covert narcissism, but although Spanish versions of instruments for self-esteem and narcissism are available, there is no available test for covert narcissism. Objective. To test the validity of the Spanish version of the Hypersensitive Narcissism Scale (HSNS) in individuals presenting drug abuse or dependence.

Method. In a sample of 79 outpatients, we assessed reliability by means of Cronbach's alpha and the intraclass correlation coefficient (ICC), construct validity through factor analysis, and concurrent validity by means of the correlation between the HSNS and measures of severity, disability, self-esteem, grandiose narcissism and personality disorders.

Results. Reliability of the HSNS total scale score was satisfactory (Cronbach's alpha $=0,73, I C C=0,67$ ), though some items would require further consideration. Factor analysis showed good construct validity with three factors compatible with the theory of covert narcissism. With regard to concurrent validity, covert narcissism (HSNS) correlated positively with open narcissism, severity and disability due to drug use, and negatively with self-esteem. Highest scores on the HSNS corresponded to borderline, narcissistic and passive-aggressive personality disorders.

Conclusions. The Spanish version of the HSNS could be a valid instrument for the assessment of covert narcissism in those treated for drug abuse or dependence.

Key words: narcissism, covert narcissism, HSNS, personality disorders, drug abuse. 


\section{INTRODUCCIÓN}

- a importancia de considerar la comorbilidad de los trastornos de personalidad en el tratamiento del abuso o dependencia de substancias ha sido ampliamente documentada tanto por su alta prevalencia como por su valor pronóstico (De Weert-Van Oene, Jorg y de Jong, 2006; Ronningstam, 1996). En población española, la prevalencia de un trastorno de personalidad asociado a un diagnóstico de uso de sustancias se estima en torno al 50-85\% (Cervera, Bolinches y Valderrama, 1999; López y Becoña, 2006a; López Torrecillas, de los Riscos Araque y de la Casa, 2004; Pedrero, Puerta, Lagares y Saez, 2003), siendo muy relevantes los trastornos del grupo B (López y Becoña, 2006a). Dado que en este grupo B se presenta un elevado solapamiento en las tasas diagnósticas entre los distintos tipos de trastornos de personalidad, para su conocimiento resulta especialmente relevante comprender la asociación de los rasgos narcisistas de personalidad con los trastornos narcisista de personalidad (TNP), trastorno antisocial (TAP) y con el trastorno límite de la personalidad (TLP). Con respecto al TNP y el TAP, ambos presentan fantasías de grandiosidad, sentimiento de invulnerabilidad, necesidad de atención, sentimientos de envidia y sensibilidad elevada a las críticas junto con un patrón de interacción personal explotador y con ausencia de empatía. (Gunderson y Roningstam, 2001). En el caso de la relación entre TNP y el TLP ambos comparten la dificultad para la regulación del afecto, la impulsividad, las relaciones inestables, sensibilidad y reacciones de rabia o rencor a las criticas (Gunderson y Ronningstam 2001; Holdwick, Hilsenroth, Castelbury y Blais, 1998; Ronningstam y Gunderson 1991). Por otro lado, aunque los pacientes que solicitan tratamiento por uso o abuso de drogas presentan una baja prevalencia de TNP, la prevalencia de rasgos narcisistas es elevada (Vaglum, 1999). Además se estima que entre el $24-50 \%$ de los sujetos con TNP presenta además trastorno por abuso de substancias, especialmente abuso de cocaína (Ronningstam, 1996).

El concepto de autoestima formó parte de la definición en 1977 del TNP de la DSM-III introduciéndose el criterio de "autoestima sobrevalorada" y definida como "...exagera los logros y capacidades o muestra una pretenciosa seguridad en sí mismo." (Millon y Davis 1998). Sin embargo en las versiones posteriores dejó de mencionarse el concepto de autoestima para hablarse entre los criterios diagnósticos del TNP de presencia de un "sentido grandioso de auto-importancia", o "creer que se es especial o único" (Millon y Davis 1998).

La autoestima carece de una definición clara y existen distintos modos de definirla variando desde el "sentimiento de valía personal" (Rosenberg, 1973) hasta la "experiencia de ser competente para afrontar los retos básicos de la vida y merecer ser feliz" (Branden, 1969). De hecho, la concepción habitual de la autoestima ha venido asumiendo que existirian dos tipos de sujetos, unos convencidos de que son excelentes y otros de que son terribles. En consonancia con esta concepción los sujetos con baja autoestima presentarían un mayor riesgo de consumo de drogas y de conducta antisocial (Heaven 1986; Vega, Zimmerman, Warheit y Aspori, 1993) e incremen- tar la autoestima sería útil para el tratamiento de consumo de drogas. Sin embargo estudios más recientes muestran en cambio que los sujetos con baja autoestima se caracterizan por no comprenderse a sí mismos de un modo consistente, de modo que pueden tener un sentimiento de grandiosidad elevado pero carecen de convicciones firmes sobre sí mismos (Campbell, 1990). Así, el consumo de substancias y las conductas antisociales serían el resultado no de una baja autoestima sino de la búsqueda de métodos para afirmar un elevado concepto de sí mismo. En una revisión de la literatura Baumeister, Campbell, Krueger y Vohs, (2003) concluyeron que una autoestima alta no protege del consumo de drogas o alcohol, sino que al contrario fomenta la experimentación llevando a un inicio temprano del consumo aunque en general los efectos de la autoestima son limitados. Por otro lado el concepto de narcisismo, sobre todo el concepto de narcisismo arrogante, recoge un importante segmento de las personas con alta autoestima y presenta mayor homogeneidad, y por ello su estudio puede resultar en hallazgos más consistentes que la autoestima (Baumeister,1999).

Entendiendo el narcisismo como una dimensión que se extendería desde el sano narcisismo en un extremo hasta el narcisismo patológico en otro extremo, dentro de este último se han distinguido por un lado el tipo de narcisismo arrogante o manifiesto y por otro el tipo vulnerable, hipersensible, vergonzoso, o encubierto (Dickinson y Pincus, 2003; Russ, Shedler, Bradley y Westen, 2008; Wink, 1991). En ambos casos se presentaría una visión sobredimensionada de sí mismo, alta sensibilidad a las críticas, egoísmo, así como deseo y búsqueda de admiración. Por un lado el narcisismo arrogante o manifiesto se caracterizaría por grandiosidad, exhibicionismo y fanfarroneria, mientras que por otro lado el narcisismo encubierto o hipersensible se caracterizaria por hipersensibilidad y vulnerabilidad en las relaciones personales (Ronningstam y Gunderson, 1991; Wink, 1991). Para la valoración del narcisismo hipersensible Hendin y Check (1997) desarrollaron un instrumento de diez items conocido como el Hypersensitive Narcissism Scale (HSNS). La escala se obtuvo en una muestra de 403 estudiantes a partir de las preguntas de la Escala de Narcisismo de Murray (1938) y del análisis previo de Wink (1991) sobre el narcisismo medido por el MMPI (Hathaway y McKlinley 1940), obteniéndose una aceptable consistencia interna, estructura factorial y validez externa. Posteriormente estos mismos autores desarrollaron otra versión ampliada de 23 ítems en fase de validación en este momento en lengua española. De entre los instrumentos disponibles para el estudio del narcisismo en nuestro ámbito se dispone de la versión española del Inventario de Personalidad Narcisista (NPI) de Raskin y Hall (1981), validado al castellano por García y Cortés (1998), sin contarse con ningún instrumento para la valoración del narcisismo hipersensible.

El objetivo de nuestro estudio es estudiar la validez de la versión española de la Escala de Hipersensibilidad Narcisista (HSNS) de 10 ítems de Hendin y Check (1997) para la evaluación de rasgos de narcisismo hipersensible en una población de usuarios de drogas en un centro ambulatorio. 


\section{MÉTODO}

\section{Muestra y diseño del estudio}

Se entrevistó a 79 pacientes drogodependientes atendidos consecutivamente en la Unidad de Conductas Adictivas Valencia 7 de la Conselleria de Sanitat de Valencia que cumplian los criterios de inclusión de edad comprendida entre 18-65 años y diagnosticados de abuso o dependencia de drogas según criterios DSM-IV, la mayor parte de ellos drogas ilegales (tabla1). Se excluyeron los pacientes con sintomatología orgánica, psicótica o afectiva que pudiera condicionar la información proporcionada. Los pacientes proporcionaron el consentimiento informado para la participación en el estudio y el diseño fue aprobado por el comité de ética del área sanitaria.

Como hipótesis se supuso una correlación positiva entre las puntuaciones de la HSNS y la gravedad de la dependencia. También se hipotetizó la ausencia de relaciones significativas entre la HSNS y las variables sociodemográficas, una correlación débil con el narcisismo manifiesto y ausencia de correlación o correlación negativa con la autoestima. Se hipotetizó también la correlación positiva entre la HSNS y una visión de sí mismo compatible con egoísmo y vulnerabilidad en las relaciones interpersonales.

\section{Instrumentos de evaluación}

Junto con las variables sociodemográficas de sexo, edad, estado civil, nivel de instrucción y situación laboral, los participantes en el estudio cumplimentaron los siguientes instrumentos:

Escala de Narcisismo Hipersensible (HSNS). La escala de narcisismo hipersensible HSNS (Hendin y Check, 1997) se obtuvo a partir de la correlación hallada entre diferentes escalas de narcisismo que los autores administraron a una población de 403 estudiantes, estableciendo 10 ítems para medir el narcisismo encubierto. La puntuación se realiza como escala tipo Likert, donde los ítems se responden en una escala de 5 puntos ( $1=$ totalmente falso, $2=$ falso, $3=$ neutro, $4=$ cierto, 5 =totalmente cierto). La puntuación total, por tanto, oscila entre 10 y 50 . En una primera fase se tradujo la HSNS al español, posteriormente se realizó una traducción inversa del español al inglés por un traductor bilingüe ciego a la versión inglesa. Esta segunda versión en inglés fue enviada a uno de los autores de la escala (Check) para su revisión, obteniéndose finalmente su aprobación.

Escala de autoestima global de Rosenberg (EAR). Se empleó la versión española de la escala de autoestima de Rosenberg (Rosenberg, 1973; Vázquez, Jiménez y Vázquez, 2004). La escala incluye 10 ítems cuyos contenidos se centran en los sentimientos de respeto y aceptación de sí mismo. La mitad de los ítems están enunciados positivamente y la otra mitad negativamente. Los items se responden en una escala de cuatro puntos ( $1=$ muy de acuerdo, $2=$ de acuerdo, $3=$ en desacuerdo, $4=$ totalmente en desacuerdo).
Escala de Personalidad Narcisista (EPN). Se empleó la versión española abreviada de 40 ítems (García y Cortés, 1998) del Narcissistic Personality Inventory (NPI) de Raskin y Hall (1981). La escala recoge los componentes de autoridad, exhibicionismo, superioridad, vanidad, explotación y autosuficiencia (Raskin y Terry, 1988). Además se mide un constructo general de narcisismo con puntuaciones altas en los sujetos extrovertidos, dominantes, exhibicionistas, agresivos, impulsivos, centrados en sí mismos, auto indulgentes, satisfechos consigo mismos y no conformistas. Esta descripción es congruente con aquellas que se esperan de manifestaciones no clínicas del narcisismo.

Diferencial semántico. El diferencial semántico es un procedimiento clásico de medición de actitudes especialmente indicado para la valoración del concepto de sí mismo (Bechini 1986), requiriendo que se elabore a propósito de cada población objeto de estudio. Para ello se siguió el método indicado por Bechini (1986). En primer lugar, siguiendo el método de selección de adjetivos de Kelly (1955), se solicitó a una muestra de usuarios de la Unidad de Conductas Adictivas que se definieran a sí mismos. De los adjetivos obtenidos se seleccionaron aquellos que coincidieron con los indicados por Bechini (1986) en una adaptación de la técnica del diferencial semántico a la lengua española. Se obtuvieron de este modo 39 pares de adjetivos opuestos (sano-enfermo, cobarde-valiente, fuerte-débil) con los que el sujeto se puntuaba a lo largo de una escala con un punto medio neutro y los polos extremos donde se situaban los adjetivos opuestos. Posteriormente se cribaron administrándose a una muestra de 20 pacientes, seleccionándose los que presentaron mayor amplitud de distribución (4 puntos) y desviación típica. Se llevó a cabo un análisis factorial que proporcionó seis factores sobre visión de sí mismo explicando el 68\% de la varianza. El primer factor resultó una percepción generalizada de Debilidad-Cobardía; el segundo factor agrupó los conceptos de Común-Corriente; el tercer factor los términos Exigencia-Agresividad; el cuarto factor los términos Dependencia-Inferioridad; el quinto factor los términos Impulsividad-Nerviosismo y finalmente, el sexto factor, agrupó el concepto de Irresponsabilidad.

Entrevista Clínica Estructurada para los Trastornos de la Personalidad del EJE II del DSM-IV (SCID II). Para la valoración de los trastornos de personalidad se empleó la versión española de la SCID-II (Gómez-Beneyto, Villar, Renovell, Pérez, Hernández, Cuquerella, et al., 1994), cumplimentando los trastornos: histriónico, narcisista, límite y antisocial junto con el trastorno pasivo-agresivo de la personalidad o trastorno negativista de la personalidad que se incluyó por razones teóricas. Dado que la relación entre algunos trastornos de personalidad y el consumo de sustancias estimulantes e ilegales es dificil de evaluar tal como requiere el SCID-II a la hora de determinar "...un patrón estable y de larga duración que se remonte a la adolescencia o al principio de la edad adulta...no debido a los efectos directos de una sustancia", tal como recomienda el manual en estos casos, se utilizó como criterio de "verdadero" la presencia de un patrón frecuente durante los últimos cinco años. 
Índice Europeo de Gravedad de la Adicción (EuropASI) (Bobes, González, Saiz y Bousoño, 1996). Se emplearon los indicadores de Situación Médica, Situación legal y Relaciones Familiares del paciente.

Escala de Discapacidad de Sheehan (SDI) (Bobes, Badía, Luque, García, Gonzalez, Dal-Ré, et al., 1999), Escala de Evaluación de la Actividad Global (EEAG; American Psychiatric Association, 2003a) y Escala de Evaluación de la Actividad Social y Laboral (EEASL; American Psychiatric Association, 2003b) del EJE V del DSM IV. Se emplearon estas tres escalas para la valoración del ajuste funcional y la gravedad de la adicción.

\section{Análisis estadístico}

Para el análisis de la fiabilidad y la validez de la HSNS se siguieron los criterios de Streiner y Norman (1989) utilizando la versión 14 del paquete estadístico SPSS.

Fiabilidad. Como medida de consistencia interna se empleó el alfa de Cronbach. La fiabilidad test-retest se determinó mediante el Coeficiente de Correlación Intraclase (CCIC) administrando la HSNS en dos ocasiones en el plazo de 15 días.

Validez. La validez de constructo se determinó mediante el análisis factorial con rotación Varimax y la validez concurrente mediante la correlación de la HSNS con la escalas de narcisismo y de autoestima así como con los índices de gravedad de la adicción. Para el estudio de la relación entre la HSNS y la visión de sí mismo se empleó el coeficiente de correlación de Pearson.

Se utilizaron la prueba t-test o el análisis de la varianza para comprobar el efecto de las variables sexo y estado civil. El posible efecto del nivel de instrucción se valoró mediante el coeficiente de correlación por rangos de Spearman.

\section{RESULTADOS}

Del total de los sujetos (79), 26 fueron mujeres (33\%) y 53 hombres $(67 \%)$. La edad media fue de $32,5($ D.T. $=6,1)$ años para las mujeres y $36,6($ D.T. $=7,3)$ años para los hom- bres (amplitud $=23-52$ ), con más años de escolarización en las mujeres $(11,5)$ que en los hombres $(10,5)$. El $81 \%$ de la muestra se encontró en situación laboral de activo. Algo más de la mitad de los pacientes estaban solteros, mientras que una cuarta parte se encontraban separados o divorciados. En cuanto a los diagnósticos de trastornos por sustancias, algo más de la mitad de la muestra tenía una dependencia de cocaína $(51,9 \%)$ y un tercio $(29,1 \%)$ dependencia de opiáceos (tabla1)

Sesenta y dos sujetos $(78,5 \%)$ de los pacientes presentaron algún trastorno de personalidad. Los más frecuentes fueron el antisocial $(34,2 \%)$ y el límite $(30,4 \%)$, y la media de trastornos de personalidad por paciente fue de 2,5. Veintinueve (37\%) pacientes presentaron un diagnóstico de trastorno de personalidad, $17(21,5 \%)$ cumplieron criterios para dos trastornos de personalidad y $16(20 \%)$ cumplieron criterios para tres o más trastornos de personalidad.

\section{Fiabilidad y consistencia interna}

El $\alpha$ de Cronbach fue de 0,73 . En la tabla 2 se muestran las correlaciones ítem-test y los coeficientes $\alpha$ de Cronbach para cada ítem. Los coeficientes de correlación intraclase entre las dos administraciones (pases) se muestran en la tabla 3.

\section{Validez}

El análisis factorial proporcionó tres factores que explicaron el 68\% de la varianza (Kaiser-Meyer-Olkin =0,67, prueba de Esfericidad de Bartlett, $\left.\chi^{2}=241, p<0,001\right)$. Los items y sus cargas factoriales se muestran en la tabla 4 . El primer factor, Hipersensibilidad, agrupa los ítems 2, 3, 6 y 7; el segundo Dificultad para Compartir (Egoísmo) agrupa los ítems 4, 5 y 10; finalmente el tercer factor Ensimismamiento (Egocentrismo) agrupa los ítems 1, 8 y 9.

Como era de esperar, el narcisismo encubierto medido por la HSNS mostró correlaciones en grado moderado y positivo con el narcisismo medido según la EPN $(r=0,44, p<0,01)$, y en grado moderado y negativo con la autoestima medida según la EAR $(r=-0,35, p<0,01)$. No se encontró correlación entre la HSNS con la edad, años escolarización y nivel de instrucción. Tampoco se apreciaron diferencias significati-

Tabla 1. Distribución de sexo por diagnóstico según criterios DSM-IV.

\begin{tabular}{|c|c|c|c|c|c|c|}
\hline \multirow[t]{2}{*}{ Diagnóstico } & \multicolumn{2}{|c|}{ Hombres } & \multicolumn{2}{|c|}{ Mujeres } & \multicolumn{2}{|c|}{ Total } \\
\hline & $\mathrm{n}$ & $\%$ & $n$ & $\%$ & $n$ & $\%$ \\
\hline Dependencia cocaina (304.20) & 30 & 56,6 & 11 & 42,3 & 41 & 51,9 \\
\hline Dependencia opiáceos (304.00) & 9 & 34,6 & 23 & 29,1 & 14 & 26,4 \\
\hline Dependencia cannabis (304.30) & 5 & 9,4 & 1 & 3,8 & 6 & 7,6 \\
\hline Dependencia alcohol (303.90) & 2 & 3,8 & 2 & 7,7 & 4 & 5,1 \\
\hline Otros diagnósticos & 2 & 3,8 & 3 & 11,5 & 5 & 6,3 \\
\hline TOTAL & 53 & 100,0 & 26 & 100,0 & 79 & 100,0 \\
\hline
\end{tabular}


Tabla 2. Consistencia interna de la HSNS.

\begin{tabular}{|c|c|c|c|c|}
\hline Item de la HSNS & Correlación item-test & Media correlación item-resto & Covarianza interitems & $\alpha$ \\
\hline $\begin{array}{l}\text { 1.- Puedo llegar a quedarme completamente enfrascado o distraido pensando en } \\
\text { mis asuntos, mi salud, mis preocupaciones o mis relaciones con los demás. }\end{array}$ & 0,56 & 0,41 & 0,23 & 0,71 \\
\hline $\begin{array}{l}\text { 2.- Mis sentimientos son fácilmente heridos por el ridiculo o comentarios criticos } \\
\text { de los demás. }\end{array}$ & 0,75 & 0,64 & 0,19 & 0,67 \\
\hline $\begin{array}{l}\text { 3.- Cuando entro en algún local me siento inseguro y siento que las miradas de } \\
\text { los demás se fijan en mi. }\end{array}$ & 0,61 & 0,46 & 0,22 & 0,71 \\
\hline 4.- Cuando tengo éxito no me gusta compartir el mérito con otros. & 0,32 & 0,16 & 0,27 & 0,75 \\
\hline 5.- Tengo bastante con mis cosas como para preocuparme por los de otra gente & 0,37 & 0,19 & 0,26 & 0,75 \\
\hline 6.- Siento que tengo un carácter distinto a la mayoría de la gente. & 0,67 & 0,53 & 0,21 & 0,69 \\
\hline 7.- Siempre interpreto de modo personal los comentarios de los demás & 0,72 & 0,61 & 0,25 & 0,68 \\
\hline $\begin{array}{l}\text { 8.- Con facilidad me meto en las cosas que me interesan y me olvido de los } \\
\text { demás. }\end{array}$ & 0,46 & 0,30 & 0,23 & 0,73 \\
\hline $\begin{array}{l}\text { 9.- Me desagrada estar con un grupo de gente a menos que sepa que uno de los } \\
\text { presentes en el grupo me aprecia. }\end{array}$ & 0,56 & 0,40 & 0,27 & 0,71 \\
\hline $\begin{array}{l}\text { 10.- En el fondo me fastidia que otra gente me venga con sus problemas, } \\
\text { pidiéndome mi tiempo y mi comprensión. }\end{array}$ & 0,33 & 0,24 & 0,27 & 0,74 \\
\hline TOTAL & & 0,23 & & 0,73 \\
\hline
\end{tabular}

Tabla 3. Coeficientes de correlación intraclase de la HSNS.

\begin{tabular}{|c|c|c|}
\hline İtem de la HSNS & Coeficientee de correlación intraclase & Intervalo de confianza \\
\hline 1 & 0,56 & $0,41-0,71$ \\
\hline 2 & 0,76 & $0,67-0,85$ \\
\hline 3 & 0,64 & $0,51-0,77$ \\
\hline 4 & 0,23 & $0,02-0,44$ \\
\hline 5 & 0,55 & $0,40-0,70$ \\
\hline 6 & 0,65 & $0,52-0,78$ \\
\hline 7 & 0,54 & $0,38-0,69$ \\
\hline 8 & 0,46 & $0,28-0,63$ \\
\hline 9 & 0,49 & $0,32-0,66$ \\
\hline 10 & 0,45 & $0,27-0,62$ \\
\hline TOTAL & 0,67 & $0,55-0,79$ \\
\hline
\end{tabular}

vas entre hombres y mujeres. Sin embargo, a diferencia de la HSNS, la EPN se correlacionó negativamente con la edad ( $r=$ $-0,27, p<0,05)$ y la EAR se correlacionó positivamente con el nivel de instrucción $(r=0,3, p<0,05)$

Respecto al concepto de sí mismo, la puntuación total de la HSNS se correlacionó positivamente con la percepción de Debilidad y Cobardía $(r=0,34, p<0,01)$, así como con el factor Exigencia y Agresividad ( $r=0,3, p<0,01)$. No se encontró correlación significativa con los factores ComúnCorriente, Inferioridad y Dependencia, Nerviosismo- Impulsividad o de Irresponsabilidad.

La HSNS se correlacionó ligeramente con la gravedad del episodio valorado según la EEAG $(r=0,26, p<0,01)$ o la EASSL $(r=0,28, p<0,01)$. La correlación con la discapacidad SDI también fue significativa para el "estrés percibido" ( $r$ $=0,22, p<0,01)$ y para el "apoyo social percibido" $(r=-0,24$, $p<0,01)$.
Respecto a los trastornos de personalidad, como se muestra en la tabla 5, los sujetos con trastorno de personalidad limite, narcisista y pasivo-agresivo presentaron mayores puntuaciones en la HSNS. Por otro lado, en caso del trastorno histriónico no se apreciaron diferencias significativas. Los sujetos con trastorno antisocial (cumpliendo criterios antes de la edad adulta y en la edad adulta) no presentaron diferencias significativas aunque rozaron la significación ( $p$ $=0,07$ ), sin embargo, los entrevistados con comportamiento antisocial solo en la edad adulta si presentaron diferencias significativas. 
Tabla 4.- Análisis factorial de la HSNS. Componentes factoriales de los factores.

\begin{tabular}{cccc}
\hline Item de la HSNS & Hipersensibilidad & Egoismo & Ensimismamiento \\
\hline 1 &, 469 &,- 228 &, 553 \\
2 &, 865 &,- 020 &, 197 \\
3 &, 782 &, 076 &,- 077 \\
4 &, 043 &, 807 &,- 065 \\
5 &, 100 &, 732 &,- 020 \\
6 &, 587 &, 223 &, 286 \\
7 &, 847 &,- 032 &, 177 \\
8 &,- 048 &, 227 &, 803 \\
9 &, 356 &,- 030 &, 596 \\
10 &,- 050 &, 747 &, 228 \\
\hline
\end{tabular}

Kaiser-Meyer-0|kin $=0,67$

Prueba de esfericidad de Bartlett $\chi^{2}=241, p<0,001$

Tabla 5. Medias de la HSNS según trastornos de personalidad.

\begin{tabular}{|c|c|c|c|c|c|c|}
\hline & \multicolumn{4}{|c|}{ Trastorno de Personalidad } & \multirow[b]{3}{*}{$\mathrm{t}^{*}$} & \multirow[b]{3}{*}{$\mathrm{p}$} \\
\hline & \multicolumn{2}{|c|}{$\mathrm{Si}$} & \multicolumn{2}{|c|}{ No } & & \\
\hline & $\mathrm{N}$ & M (D.T.) & $\mathrm{N}$ & M (D.T.) & & \\
\hline Narcisista & 10 & $31,6(6,8)$ & 69 & $26,9(4,9)$ & $-2,71$ & $<0,01$ \\
\hline Limite & 24 & $29,5(5,7)$ & 55 & $26,6(5,0)$ & $-2, .0$ & $<0,05$ \\
\hline Histriónico & 14 & $27,7(5,8)$ & 65 & $27,5(5,3)$ & $-0,18$ & ns \\
\hline Pasivo-agresivo & 15 & $30,7(4,5)$ & 64 & $26,8(5,2)$ & $-2,64$ & $<0,01$ \\
\hline Antisocial & 27 & $29,0(5,6)$ & 52 & $27,1(5.1)$ & $-1,8$ & ns \\
\hline Comportamiento Antisocial Adulto & 55 & $28,4(5,4)$ & 24 & $25,3(4,7)$ & $-2,43$ & $<0,05$ \\
\hline
\end{tabular}

*Prueba t-test

\section{DISCUSION}

El objetivo de este estudio ha sido evaluar las características psicométricas de la HSNS, en relación con su fiabilidad y validez, adaptada en una población española de pacientes atendidos en una Unidad de Conductas Adictivas. De los 79 entrevistados, 63 cumplieron criterios de trastorno de personalidad. Si bien la prevalencia de los trastornos de personalidad encontrados en nuestra muestra podría parecer elevada, esta cifra se encuentra en el intervalo de prevalencias entre el $47 \%$ y el $97 \%$ obtenidas en diversos estudios de trastornos de personalidad en poblaciones de sujetos en tratamiento por abuso o dependencia de sustancias (López y Becoña, 2006b). Estudios Ilevados a cabo con el SCID-II en poblaciones similares a la nuestra han encontrado prevalencias semejantes (Weiss, Mirin, Griffin, Gunderson y Hufford 1993) o incluso superiores (Bricolo, Gomma, Bertani y Serpelloni, 2002; Sánchez, Tomás y Climent, 1999) a las de nuestra muestra. De todos modos, la variabilidad de las características de las poblaciones atendidas en los diversos centros de atención a drogas así como los distintos procedimientos de evaluación para determinar la presencia de un criterio de trastorno de personalidad, limitan la validez externa de este trabajo y serán necesarios estudios con poblaciones más amplias y diversas en el futuro. En este sentido es conveniente recordar que por muy extensa que sea la población donde se valida una prueba, en sentido estricto no se valida el instrumento sino su uso en el contexto de la población en el que se ha utilizado. Con ello se determina el grado de confianza que podemos hacer de las inferencias realizadas sobre los sujetos a los que se ha administrado la escala (Streiner y Norman 1989, p.108).

La consistencia interna del HSNS fue satisfactoria ( $\alpha=$ 0,73 ) en consonancia con el estudio original en lengua inglesa que presentó resultados semejantes $(\alpha=0,72)$. Los ítems de la escala mostraron una correlación aceptable con el resto de la escala excepto los ítems 4, 5 y 10 que mostraron una correlación más débil. Estos tres items forman el factor Dificultad para Compartir o Egoísmo, por lo que podría considerarse como una dimensión distinta aunque relacionada con los otros dos factores Hipersensibilidad y Ensimismamiento. La escala precisaría incrementar el número de ítems para mejorar su consistencia interna, sobre todo en lo que respecta a este factor Egoísmo-Dificultad para Compartir.

La fiabilidad test-retest también fue aceptable ( CCIC $=$ $0,67)$, con un solo ítem con nivel bajo de correlación aunque estadisticamente significativa ( $\mathrm{CCIC}=0,23$, IC 95\% = $0,02-0,44)$. El ítem corresponde a la afirmación "cuando tengo éxito no me gusta compartirlo con los demás". Posibles factores relacionados con la estabilidad de la percepción de éxito personal en esta población con escaso éxito socialmente reconocido podrian explicar las diferencias de este ítem con respecto a los otros. 
Con respecto a la validez de constructo, el instrumento presentó también una aceptable estructura factorial según indican las pruebas de K-M-O y de esfericidad de Bartlett. Además, destaca la presencia de tres factores diferenciados que reflejan claramente las tres dimensiones teóricas del narcisismo encubierto o hipersensible. Con respecto a la validez concurrente, la correlación moderada con el narcisismo medida por la escala NPI y la correlación también moderada pero negativa con la autoestima indican que, en consonancia con la teoría, el narcisismo hipersensible es un rasgo relacionado con el narcisismo pero caracterizado por baja autoestima. También en consonancia con la teoría, el sujeto con narcisismo hipersensible presenta una visión de sí mismo caracterizada por una percepción de debilidad y cobardia, así como exigente y hostil.

Resalta la ausencia de relación de la escala con variables sociodemográficas como el género, la edad y el nivel de instrucción, indicando su independencia de factores sociodemográficos asociados a clase o rol social. En los trastornos de personalidad relacionados teóricamente con el narcisismo ostentoso (trastorno histriónico) o con el narcisismo psicópata (trastorno antisocial) no se han encontrado diferencias significativas en las puntuaciones del HSNS. Este hallazgo es congruente con la teoría que indica la existencia de un tercer tipo distinto de narcisismo. Por el contrario los trastornos narcisista, pasivo agresivo y límite presentan un componente narcisista hipersensible todos ellos con el factor común de su alta sensibilidad a las críticas o al rechazo. El narcisismo hipersensible se relacionó con el comportamiento antisocial adulto, pero no se relacionó con el trastorno antisocial desde la infancia. En este sentido, parece claro que se trata de un constructo que refleja el ensimismamiento así como la disposición al egoísmo pero es independiente de la ausencia de empatía y de la sensibilidad al sufrimiento de los demás.

En conclusión, el presente estudio ha aportado datos sobre la validez y la fiabilidad de la escala de narcisismo hipersensible, Hypersensitive Narcissism Scale (HSNS), sugiriendo una aceptable consistencia interna así como fiabilidad, validez factorial y validez externa. De todos modos se precisarán estudios con muestras mayores para mejorar algunos de sus items. El narcisismo encubierto o hipersensible puede resultar un constructo útil en la práctica clíni$c a$, ya que mide una dimensión presente en trastornos de personalidad importantes en el pronóstico y tratamiento de las conductas adictivas como el trastorno límite, narcisista, pasivo-agresivo o el comportamiento antisocial, siendo independiente de otros tipos de narcisismo, como el narcisismo ostentoso reflejado en el trastorno narcisista de la personalidad o en el trastorno antisocial de la personalidad.

\section{AGRADECIMIENTOS}

Al apoyo y supervisión proporcionado por la Asociación Valenciana para la Investigación y Docencia de la Salud Mental.

\section{REFERENCIAS}

American Psychiatric Association. (2003a). Escala de evaluación de la actividad global (EEAG). En: Manual diagnóstico y estadístico de los trastornos mentales DSM-IV-TR (pp.40). Barcelona: Masson.

American Psychiatric Association. (2003b). Escala de evaluación de la actividad social y laboral (EEASL). En: Manual diagnóstico y estadistico de los trastornos mentales DSM-IV-TR (pp. 910-911). Barcelona: Masson.

Baumeister, R. F. (1999). The self in social psychology (pp. 220-221). Philadelphia: Taylor \& Francis.

Baumeister, R. F., Campbell, J. D., Kreuger, J. I. y Vohs, K. D. (2003). Does high self-esteem cause better performance, interpersonal success, happiness or healthier lifestyles? Psychological Science in the Public Interest, 4(1), 1-44.

Bechini A. (1986). El diferencial semántico. Barcelona: Hispano Europea.

Bobes, J., González, M. P., Sáiz, P. A., Bousoño, M. (1996). Indice Europeo de severidad de la adicción: EuropASI. Versión española. En: Libro de Actas IV Reunión Interregional de Psiquiatría (pp.201-218).

Bobes, J., Badia, X., Luque A., Garcia M., González M. P., Dal-Ré, R. y Grupo de Validación en Español de Cuestionarios de la Fobia Social. (1999). Validación de las versiones en español de los cuestionarios Liebowitz Social Anxiety Scale, Social Anxiety and Distress Scale y Sheehan Disability Inventory para la evaluación de la fobia social. Medicina Clínica, 112, 530-538.

Branden N. (1969). The Psychology of Self-Esteem. New York: Ban Tam. Bricolo, F., Gomma, M., Bertani, M. y Serpelloni, G. (2002). Prevalencia de trastornos de personalidad en una muestra de 115 clientes con trastornos por uso de drogas. Adicciones, 14, 491-496

Campbell, J. (1990). Self-esteem and clarity of the self-concept. Journal of Personality and Social Psychology, 59, 538-549.

Cervera, G., Bolinches, F. y Valderrama, J. C. (1999). Trastornos de personalidad y drogodependencias. Trastornos Adictivos, 1 , 91-9.

De Weert-Van Oene, G. H., Jorg, F. y De Jong, C. A. (2006). Association between interpersonal behaviour and helping alliance in substance-dependent patients. European Addiction Research, 12, 67-73.

Dickinson, K. A. y Pincus, A. C. ( 2003). Interpersonal análisis of grandiose and vulnerable narcissism. Journal of Personality Disorders, 17,188-207

Garcia, J. M. y Cortés, J. F. (1998). La medición empírica del narcisismo Psicothema, 10, 725-735.

Gómez-Beneyto, M., Villar, M., Renovell, M., Pérez, F., Hernández, M., Cuquerella, M., et al. (1994). The diagnosis of Personality disorders with a modified version of the SCID-II in a spanish clinical sample. Journal of Personality Disorders, 8, 104-110.

Gunderson, J. y Ronningstam, E. (2001). Differentiating antisocial and narcissistic personality disorder. Journal of Personality Disorders, 15, 103-109

Hathaway, S. R. y McKinley, J. C. (1940). A multiphasic personality schedule. Construction of the schedule. Journal of Psychology, 10, 249-254.

Heaven, P. (1986). Correlates of conformity in three cultures. Journal of Personality and Social Psychology, 54, 883-887. 
Hending, H. y Check, J. (1997) Assessing Hypersensitive Narcissism: A Reexamination of Murray's Narcissism Scale. Journal of Research in Personality, 31, 588-599.

Holdwick, D., Hilsenroth, M., Castelbury, F. D. y Blais, M. (1998). Identifying the unique and common characteristics among the DSM-IV antisocial, borderline and narcissistic personality disorder. Comprhensive Psychiatry, 39, 227-286

Hendin, H. M. y Cheek, J. M. (1997). Assessing Hypersensitive Narcissism: A Re-examination of Murray's Narcissism Scale. Journal of Research in Personality, 31, 588-599.

Kelly, GA. (1955). The psychology of personal constructs. New York: Norton.

López Durán, A. y Becoña Iglesias, E. (2006a). Patrones y trastornos de personalidad en personas con dependencia de la cocaina en tratamiento. Psicothema, 18, 578-83.

Lopez Duran, A. y Becoña Iglesias, E. (2006b). Consumo de cocaina y psicopatología asociada: una revisión. Adicciones, 18, 161-196.

López Torrecillas, F., De los Riscos, M., Araque, F. y de la Casa L. (2004). Trastornos de personalidad: una comparación entre consumidores de cocaina, heroína y alcohol. Revista Española de Drogodependencias, 3-4, 224-237.

Millon T, Davis R D. (1998). Trastornos de la personalidad: más allá del DSM-IV. Barcelona: Masson.

Murray, H. (1938). Explorations in personality. New York: Oxford University Press.

Pedrero E., Puerta, C., Lagares, A. y Sáez, A. (2003). Prevalencia en intensidad de trastornos de personalidad en adictos a sustancias en tratamiento en un centro de atención a las drogodependencias. Trastornos Adictivos, 5, 241-55.

Raskin, R. y Hall, C. S. (1981). The Narcissistic Personality Inventory: Alternate form reliability and further evidence of construct validity. Journal of Personality Assesment, 45, 159-162.

Raskin R. y Terry, H. (1988). A Principal-Components Analysis of the Narcissistic Personality Inventory and Further Evidence of Its
Construct Validity. Journal of Personality and Social Psychology, 54, 890-902.

Ronningstam, E. y Gunderson, J. (1991). Differentiating borderline personality disorder from narcissistic personality disorder. Journal of Personality Disorder, 5, 225-232

Ronningstam, E. (1996). Patological Narcissism and Narcissistic Personality Disorder in Axis I Disorders. Harvard Review of Psychiatry, 3, 326-340.

Rosenberg, M. (1973). La autoimagen del adolescente en la sociedad. Buenos Aires: Paidós.

Rus, E., Shedler, J., Bradley, R., y Westen, D. (2008). Refining the construct of narcisistic personality disorder: diagnostic and criteria subtypes. American Journal of Psychiatry, 165, 14731481.

Sanchez, E., Tomás, V., Climent, A. (1999). Trastornos de personalidad en adictos a opiáceos. Adicciones, 3, 221-227.

Streiner, D., y Norman, G. (1989). Health Measurement Scales. A practical guide to their development and use. Oxford. Oxford University Press.

Vaglum, P. (1999). The narcissistic personality disorder and addiction. In J Derksen, Maffei C \& Groen (Eds). Treatment of personality disorders (pp. 241-253). New York: Kluwer Academic/Plenum.

Vázquez Morejón, A. J., Jiménez Garcia-Bóveda, R., y VázquezMorejón, R. (2004). Escala de autoestima de Rosenberg: fiabilidad y validez en población clínica española. Apuntes de psicología, 22(2), 247-255.

Vega, W., Zimmerman, R., Warheit, G. y Apospori, E. (1993). Risk factors for early adolescent drug use in four ethnic and racial groups. American Journal of Public Health, 83, 185-189.

Weiss, R. D., Mirin, S. M., Griffin, M. L., Gunderson, J. G. y Hufford, C. (1993). Psychopathology in cocaine abusers. The Journal of Nervous and Mental Disease, 176, 719-725

Wink, P. (1991). Two faces of narcissism. Journal of Personality and Social Psychology, 61, 590-597. 